\title{
Des lieux de lecture : de la littérature aux activités scolaires
}

Jean-François Massol

\section{OpenEdition}

1 Journals

Édition électronique

URL : http://journals.openedition.org/recherchestravaux/668

DOI : 10.4000/recherchestravaux.668

ISSN : 1969-6434

Éditeur

UGA Éditions/Université Grenoble Alpes

Édition imprimée

Date de publication : 31 décembre 2013

Pagination : $57-75$

ISBN : 978-2-84310-267-7

ISSN : 0151-1874

Référence électronique

Jean-François Massol, « Des lieux de lecture : de la littérature aux activités scolaires », Recherches \& Travaux [En ligne], 83 | 2013, mis en ligne le 01 juillet 2015, consulté le 08 septembre 2020. URL

http://journals.openedition.org/recherchestravaux/668; DOI : https://doi.org/10.4000/ recherchestravaux.668 
Jean-François Massol

Université Grenoble Alpes, EA Traverses I9-2I ${ }^{1}$

\section{Des lieux de lecture : de la littérature aux activités scolaires}

Une main pendante, et l'autre soutenant son front, le jeune Aristote, assis sur un siège rembourré et les pieds confortablement croisés, lit, alangui, un papyrus déroulé sur ses genoux. [...] Assis sur une marche spacieuse, le menton reposant sur le bout des doigts de la main droite, Saint Dominique, absorbé dans le livre qu'il tient ouvert sur ses genoux, semble sourd au monde. Blottis sous un arbre, deux amants, Paolo et Francesca, lisent un vers d'un poème qui leur sera fatal [...] En chemin vers l'école de médecine, deux étudiants islamiques du XII siècle s'arrêtent pour consulter un passage d'un des livres qu'ils portent. Le doigt pointé sur la page de droite d'un livre ouvert sur ses genoux, l'Enfant Jésus explique sa lecture aux Anciens réunis dans le Temple tandis qu'eux, étonnés et sceptiques, tournent en vain les pages de leurs volumes respectifs à la recherche d'une réfutation $[\ldots]^{2}$

Décrivant d'une ou deux phrases, dans l'incipit de son Histoire de la lecture, dixhuit représentations imagées de lecteurs célèbres ou anonymes, Alberto Manguel témoigne de la dimension concrète d'une activité mentale qui implique une posture, en un lieu plus ou moins précis, dans la solitude ou avec d'autres. Alberto Manguel en conclut qu'il n'est pas seul.

Lui, eux, nous, qui lisons, le faisons en de nombreux lieux, en des lieux très divers.

La chambre est un lieu fréquent, lieu banal, lieu que la lecture peut «néantiser» comme le suggère Simone de Beauvoir ${ }^{3}$. Mais on lit en bien d'autres pièces de la demeure, de la maison ou de l'appartement, dans la salle à manger

I. Centre de recherche en didactique de la littérature (CEDILIT).

2. A. Manguel, Histoire de la lecture, trad. C. Le Bouf, Arles, Actes Sud, 1998.

3. "Je tire les rideaux de ma chambre, je m'étends sur un divan, tout décor est aboli, je m'ignore moi-même : seule existe la page noire et blanche que parcourt mon regard.» (Tout compte fait, Paris, Gallimard, 1972, p. 158 ) 
comme le fait, le matin, le narrateur proustien de "Journées de lecture ${ }^{4}$ », dans la bibliothèque du grand-père comme Jean-Paul Sartre enfant ${ }^{5}$, dans l'antichambre comme c'est le cas pour Simone de Beauvoir dans son enfance, dans une "petite lanterne en bois naturel, qui a l'air d'une dunette de navire, et qui surplombe comme un poste de vigie un hall obscur et profond" comme, à la villa Montmorency, André Gide lisant des pages de Si le grain ne meurt à Roger Martin du Gard ${ }^{7}$, ou encore dans un "petit cabinet sentant l'iris ${ }^{8}$ " pour ce qui est encore du Narrateur proustien, celui de La Recherche du temps perdu cette fois. On peut élargir la liste, avec les chambres d'hôtel comme celle où Alberto Manguel lit un roman canadien pour enfants ${ }^{9}$; mais aussi ces hôtels un peu particuliers que sont les prisons ${ }^{\mathrm{TO}}$; ou encore une cabane perdue dans les bois ${ }^{\mathrm{II}}$. On lit aussi à l'extérieur, dans le jardin par exemple, privé mais aussi public ${ }^{12}$; dans les moyens de transport, dans le train comme le rappelle Simone de Beauvoir ${ }^{13}$ encore, sur un «petit huilier» africain comme c'est le cas pour Gide ${ }^{14}$,

4. M. Proust, «Journées de lecture», dans Pastiches et mélanges, Contre Sainte-Beuve, éd. P. Clarac et Y. Sandre, Paris, Gallimard, I97I, coll. «Bibliothèque de la Pléiade», p. I6I.

5. J.-P. Sartre, Les Mots, Paris, Gallimard, 1964.

6. Dans cette antichambre, S. de Beauvoir s'installait «en face de l'armoire normande et de l'horloge en bois sculpté qui enfermait dans son ventre deux pommes de pin cuivrées et les ténèbres du temps; dans le mur s'ouvrait la bouche d'un calorifère; à travers le treillis doré je respirais un souffle nauséabond qui montait des abîmes. Ce gouffre, ce silence, scandés par le tic-tac de l'horloge m'intimidaient. Les livres me rassuraient.» (Mémoires d'une jeune fille rangée, Paris, Gallimard, 1958, p. 70)

7. R. Martin du Gard, 6 octobre I920, Journal, t. 2, éd. C. Sicard, Paris, Gallimard, I993, p. 170.

8. M. Proust, Du côté de chez Swann, éd. J.-Y. Tadié, Paris, Gallimard, 1987, coll. «Bibliothèque de la Pléiade", p. I2-I3.

9. "En décembre, il y a deux hivers, en attendant, dans le Grand Hôtel de Poitiers, que les propriétaires de la maison conviennent d'une date pour la signature, je lisais Le Vent dans les saules.» (A. Manguel, Journal d'un lecteur, trad. C. Le Bøuf, Arles, Actes Sud, 2006, p. I44)

Io. A. Gramsci, Lettres de prison (I926-1934) [en ligne], trad. H. Albani, C. Depuyper et G. Saro, Paris, Gallimard, coll. "Témoins». Disponible sur $<$ http://classiques.uqac.ca/classiques/gramsci_antonio/lettres_de_prison/lettres_prison_avert.html> [consulté le I5/07/2013], Passion.

II. S. Tesson, Dans les forêts de Sibérie, Paris, Gallimard, 20II, passim.

I2. Dans les Mémoires d'une jeune fille rangée, ouvr. cité, S. de Beauvoir évoque à la fois le jardin familial de Meyrignac et le jardin du Luxembourg.

I3. «Il m’est agréable aussi de lire dans un train. Mon regard reçoit avec une quasi-passivité les paysages qui défilent derrière la vitre, il revient parcourir le texte auquel il prête vie.» (S. de Beauvoir, Tout compte fait, ouvr. cité, p. I59)

I4. «Le Ruby est flanqué de deux baleinières aussi longues que lui, chargées de bois, de caisses et de nègres. Il fait frais, moite et terriblement orageux. [...] Relu l'oraison funèbre de Marie-Thérèse d'Autriche. Admirables passages; je crois bien que je la préfere à celles des deux Henriettes. (A. Gide, Voyage au Congo, Paris, Gallimard, 1927, coll. "Idées Gallimard», p. 44) 
dans l'avion comme Alberto Manguel encore ${ }^{\mathrm{I}}$. On a lu dans «une voiture fermée tirée par un cheval ${ }^{16}{ }^{\prime}$, on doit pouvoir lire dans cette forme particulière de voiture tirée par des chevaux qu'est un carrosse ${ }^{17}$. On lit au café, comme le fait Antoine Thibault lorsqu'il prend connaissance de la nouvelle écrite par son frère Jacques ${ }^{18}$, dans une bibliothèque, qu'elle soit personnelle comme celle de Montaigne ou celle que s'est fait construire Alberto Manguel ${ }^{19}$, ou publique comme celle que fréquente l'Autodidacte sartrien ${ }^{20}$, ou encore celle du duc de Guermantes $^{21}$. On lit dans une étude que la ferveur mise à la découverte de Robinson Crusoë fait oublier ${ }^{22}$, ou en bravant les interdits ${ }^{23}$, on lit en classe, par exemple en cours d'histoire en glissant Le Bruit et la Fureur de Faulkner «sur (ses) genoux, sous le pupitre ${ }^{24} »$. On lit sur la plage ${ }^{25}$, on lira ou on se promet de lire sur une île déserte ${ }^{26} \ldots$

La multiplicité et la diversité des lieux de lecture ne constituent pas le tout de cette question. Celle-ci recouvre plusieurs autres dimensions. Le lieu vaut parfois pour le contexte même de la lecture, dans sa matérialité, sa concrétude : la lecture est, à sa façon, une activité physique. Le lieu s'inscrit ainsi dans la dimension spatiale de la lecture, moins simple qu'il n'y paraît à première vue et sur laquelle je reviendrai. Le lieu de lecture peut aussi impliquer une fonction :

I5. «Dans l'avion, je sors un exemplaire de L'Invention de Morel, d'Adolfo Bioy Casares, l'histoire d'un homme échoué sur une île apparemment habitée par des fantômes..." (Journal d'un lecteur, ouvr. cité, p. I9)

I6. N. Sarraute, Enfance, Paris, Gallimard, 1983, coll. «Folio», p. 8I.

I7. A. Bennet, La Reine des lectrices, trad. P. Ménard, Paris, Denoël, 2009, p. 48-52.

I8. R. Martin du Gard, La Sorellina, dans Euvres complètes, t. I, Paris, Gallimard, I955, coll. "Bibliothèque de La Pléiade", p. II72-II92.

19. «Pendant la journée, la bibliothèque est un royaume d'ordre. D'un bout à l'autre des passages identifiés par les lettres de l'alphabet, je me déplace avec une intention manifeste, à la recherche d'un nom ou d'une voix, convoquant les livres en fonction du rang et du classement qui leur sont alloués. [...] Mais, la nuit, l'atmosphère change. Les bruits sont étouffés, les pensées plus sonores. "C'est seulement lorsqu'il fait noir que la chouette de Minerve prend son envol.» (A. Manguel, La Bibliothèque, la nuit, Arles, Actes Sud, 2009, p. 25)

20. J.-P. Sartre, Paris, La Nausée, Paris, Gallimard, 1938.

2I. M. Proust, À la Recherche du temps perdu. Le Temps retrouvé, Paris, Gallimard, coll. «Bibliothèque de la Pléiade», I954.

22. J. Vallès, L'Enfant, dans Euvres, t. II, I87I-I88I, éd. R. Bellet, Paris, Gallimard, coll. "Bibliothèque de la Pléiade», I990, p. 2IO-2II.

23. R. Martin du Gard, Le Cahier gris, dans CEuvres complètes, t. I , ouvr. cité, p. 584.

24. A. Desarthe, Comment j'ai appris à lire, Paris, Stock, 2013, p. 89.

25. "Lecture et farniente sur la plage», dans M. Proust, Jean Santeuil, précédé de Les Plaisirs et les jours, éd. P. Clarac, Paris, Gallimard, 197I, coll. «Bibliothèque de la Pléiade», p. 366-370. 26. "La première fois que j'ai lu L'Île du docteur Moreau, c'était pendant les vacances d'été. J'avais douze ans. Le livre était un cadeau d'anniversaire de mon meilleur ami, Lenny Fagin. [...] Welles devait désormais s'ajouter à ma provision pour l'île déserte.» (A. Manguel, Journal de lecture, ouvr. cité p. 44) 
lire seul dans sa chambre ou dans un jardin est lire pour soi, alors que le faire au Temple de Jérusalem ou dans une classe est lire avec d'autres ou pour les autres. Et puis le lieu de lecture peut aussi jouer le rôle d'un marqueur, comme dans certaines des questions que posent aux individus qu'ils interrogent les sociologues de la lecture : déclarer si on lit "sur son lit», à un bureau, à la bibliothèque, s'ajoute à l'estimation du nombre de livres lus dans telle ou telle période et à d'autres critères pour différencier entre eux les lecteurs ${ }^{27}$.

Dans le cadre de la réflexion sur la lecture subjective qui nous intéresse, ce foisonnement des lieux a trouvé une théorisation par Michel Picard. Choisissant de penser la lecture de la littérature en définissant celle-ci comme jeu, Michel Picard reprend à Freud et à Donald Winnicott la notion d'aire transitionnelle. Menant sa lecture dans la réalité concrète d'un lieu du monde mais reprenant inconsciemment les activités qui lui ont permis d'apprivoiser le départ angoissant de la mère, le lecteur investit de fait un espace ludique et se dédouble : il est à la fois "du côté de l'abandon, des pulsions plus ou moins sublimées» et "du côté du réel, les pieds sur terre ${ }^{28}$ ». Michel Picard poursuit :

en dépit du caractère un peu hypnotique de la situation, n'oublions jamais que le champ visuel du liseur embrasse nécessairement une étendue qui excède très largement la surface du livre ouvert; que, comme pour l'illusionniste de grande classe, il «voit les mains», qui manipulent les pages dont il entend le léger bruit, il pèse le poids du livre dans les doigts, ou sur les genoux, ou sur la poitrine, il sent l'odeur du papier, de la colle, de l'encre, il touche la couverture (de carton, de plastique, de toile, de cuir)...

Cependant, de cette grande présence des lieux de lecture dans les représentations imagées de lecteurs et chez les écrivains, de cette reconnaissance de la dimension physique de la lecture dans la théorisation qu'en propose Michel Picard, il ne reste soudain plus rien dans la réflexion menée par Vincent Jouve dans L'Effet-personnage dans le roman ${ }^{29}$, une opération de minimisation qui se trouve reprise dans La Lecture du même auteur ${ }^{30}$. Retenant de Michel Picard

27. Dans le compte rendu de l'enquête menée par C. Baudelot, M. Cartier et C. Detrez, Et pourtant ils lisent, Paris, Seuil, I999, la question du lieu proprement dit est liée à celle de la "posture corporelle» comme indicateur d'un type de lecture : "La prégnance de cette lecture divertissante se retrouve dans la posture corporelle systématiquement associée à la lecture. Pour les lecteurs occasionnels comme pour les lecteurs réguliers, la posture principale est "allongé sur le lit". Isabelle, fille de cadres intellectuels, est la seule à avoir mentionné une autre posture, "assise au bureau", à propos des livres lus pour l'école. D’autres élèves déclarent au contraire, sur le ton de l'évidence, lire toujours sur leur lit, même les livres pour l'école. Lire est donc associé à la détente et au relâchement du corps" (p. I43).

28. M. Picard, La Lecture comme jeu, Paris, Minuit, I986, p. II2-II3.

29. V. Jouve, L'Effet-personnage dans le roman, Paris, Presses universitaires de France, 1992.

30. V. Jouve, La Lecture, Paris, Hachette, coll. «Contours littéraires», I993. 
la théorisation des trois instances du lecteur empirique, Vincent Jouve a proposé, en effet, d'abandonner le «liseur » qui se trouvait distingué du «lu» puis du «lectant»:

Si la notion de lectant nous semble riche et pertinente, nous renoncerons au concept de liseur (peu opératoire) et nous détacherons du concept de lu celui de lisant (part du lecteur piégée par l'illusion référentielle et considérant, le temps de la lecture, le monde du texte comme un monde existant) $)^{31}$.

Cet abandon est, on le voit, réalisé de manière abrupte et sans justification développée, même si les analyses qui sont menées ensuite sur le «lu», le «lectant» et le «lisant» montrent que ce qui intéresse Vincent Jouve, ce sont les opérations mentales en jeu dans l'activité, nullement ses circonstances, de fait considérées comme nulles et sans intérêt. En regard et dans une perspective didactique, on peut évoquer différents lieux pensés dans l'institution scolaire, les «coins lecture» organisés dans les classes de maternelle ou de l'école primaire, la prise en compte des lectures "à la maison» dans les plans de travail définis par les professeurs de collège, de lycée, ou même les lectures à faire en bibliothèque, quand on pense à l'université.

De la multiplicité des lieux de lecture à leur organisation schématisée et à leur abandon théorique soudain, il y a un ensemble de points de vue étonnant, qui pose question. Dans le cadre de la réflexion didactique sur le sujet lecteur-scripteur a-t-on intérêt à laisser de côté la question des lieux de lecture comme le fait Vincent Jouve, ou est-il intéressant de penser cette dimension?

Pour répondre à cette question, je m'intéresserai aux lieux de lecture dans l'œuvre de Marcel Proust. Si, en effet, de nombreux auteurs mentionnent les lieux où ils ont lu ou bien ceux où ils installent momentanément leurs personnages occupés à cette activité, comme le montrent les exemples relevés supra, le texte intitulé «Journées de lecture " évoque magnifiquement, avec une profusion de détails, différents lieux des lectures d'enfance et, pour cela, il sert souvent de référence ${ }^{32}$. Préface à la traduction de deux conférences de Ruskin, Sésame et les lys ${ }^{33}$ la première publication de «ce texte capital» qui «contient

3I. V. Jouve, L'Effet-personnage dans le roman, ouvr. cité, p. 8I.

32. Par exemple, dans l'avant-propos du recueil réunissant les communications du premier colloque consacré au sujet lecteur, dans une perspective littéraire et didactique : A. Rouxel et G. Langlade, Le Sujet lecteur, lecture subjective et enseignement de la littérature, Rennes, Presses universitaires de Rennes, 2004, p. II.

33. Un autre texte reprend le même titre, mais au pluriel. Il s'agit d'un article publié en mars 1907 dans Le Figaro: M. Proust, Journées de lecture, éd. établie et présentée par A. Coelho, Paris, Union générale d'éditions, I993, coll. «IO-I8», p. 59-66. Ce texte qui évoque les lectures obligées des mondains en cas d'épidémie et quand ils ont épuisé les plaisirs des conversations téléphoniques, ne mentionne qu’un seul lieu de lecture, le "chez soi.». 
"Combray" en germe ${ }^{34}$ » en a été faite dans la revue La Renaissance latine en 1905; il a été repris dans les Mélanges en I919, mais de nombreuses publications postérieures et récentes le constituent en œuvre autonome ${ }^{35}$ même si des références à la pensée de Ruskin à laquelle il sert d'introduction sont présentes. Ce très beau texte n'est cependant pas le tout de la pensée que mène Proust sur la lecture, une thématique de son œuvre à considérer avec attention ${ }^{36}$. En tout cas, pour ce qui est des conceptions de la lecture littéraire, le privilège souvent donné à cette représentation tend à installer subrepticement des oppositions non dites qu'il vaut peut-être mieux ne pas entériner si l'on a pour objectif de développer l'intérêt de tous les élèves pour la lecture littéraire. Des analyses que je vais mener à partir de l'œuvre de Proust, je me propose de tirer in fine quelques pistes de réflexion sur les lieux de la lecture scolaire.

\section{Diversité relative et complexe des lieux de lecture dans l'œuvre de Marcel Proust}

Amateur déclaré de beaux vers, se représentant comme lecteur captivé et heureux du Capitaine Fracasse dans son enfance, pastichant plus tard Balzac, Flaubert, Régnier, Les Goncourt, Michelet, Faguet, Renan, Saint Simon, théorisant une approche de la littérature "contre Sainte-Beuve» qui renouvelle l'approche critique, grand critique lui-même, Marcel Proust est un lecteur cultivé, bien sûr ${ }^{37}$. Mais ce sont seulement les lectures d'enfance qui l'amènent à représenter la lecture de livres et le contexte de celles-ci, les œuvres et les auteurs auxquels il fait référence par ailleurs comme adulte étant, en effet, l'objet de discussions, de développements critiques sans que le moment de leur lecture soit représenté ou évoqué.

34. J.-Y. Tadié, Marcel Proust, Paris, Gallimard, 1996, p. 577.

35. Sous le titre Journées de lecture, on peut ainsi relever une édition chez publie.net en 20IO; une autre précédée d'une préface de P. Bergounioux, chez Fata Morgana en 2006; une troisième en collection Io/I8, en I993; une dernière qui suit un volume Sur la lecture, chez Librio en I984.

36. "La lecture est un motif fondamental de la Recherche, un nœud de significations. Toutefois, il faut prévenir une tendance de la critique proustienne qui l'apparente trop souvent à l'apologétique, la tentation de faire de la lecture comme thème en effet central, ce lieu commun : Marcel Proust fut un grand lecteur [...] il importe de ne pas confondre l'importance, qualitative, de la lecture pour Proust (pour l'écriture proustienne) et l'importance, quantitative, de la lecture de Proust et de l'intertexte de la Recherche." (A. Compagnon, La Troisième République des Lettres. De Flaubert à Proust, Paris, Seuil, 1983, p. 223)

37. Pour A. Compagnon, il a «la culture de la bourgeoisie cultivée au tournant du siècle» (ibid., p. 223). 
Considéré en lui-même dans un premier temps, le texte intitulé «Journées de lecture» envisage d'abord les lectures d'enfance dans une perspective narrative, avant d'évoquer et discuter plusieurs dimensions de la lecture, comme incitation, comme conversation avec des esprits supérieurs, comme thérapie, comme amitié, mais aussi comme activité dangereuse parce que ne constituant pas une réflexion personnelle. De fait, ces deux moments évoquent successivement deux formes de lecture : les lectures d'enfance sont des lectures de romans, d'un seul roman d'ailleurs, Le Capitaine Fracasse de Théophile Gautier; la lecture analysée dans différentes dimensions est une lecture de réflexion, plutôt du côté de l'adulte. Dans la perspective du sujet lecteur, plusieurs éléments de ce texte sont intéressants : dans la deuxième partie, par exemple, les bienfaits de la lecture pour certains esprits malades correspondent aux fonctions que Michèle Petit a observées dans des populations en difficultée ${ }^{38}$. Les lectures d'enfance évoquées initialement sont des lectures individuelles, à propos desquelles le jeune lecteur est représenté dans sa relation forte aux personnages qu'il aime comme des personnes réelles. En outre et surtout, le long développement initial met en évidence l'importance du contexte le plus concret, en particulier celle des lieux de lecture qui sont soit des refuges permettant d'abriter le jeune lecteur pour lui permettre de continuer à lire malgré tous les obstacles que lui opposent, le plus souvent pour son bien, les membres de sa famille les plus attentionnés, soit des causes d'arrêt lorsque le bruit de l'eau d'une pompe lui fait «lever les yeux ${ }^{39}$ ». On est ainsi du côté de la lecture comme repli sur soi, comme retrait.

Décrits avec une précision relative mais magnifiquement évocatoire, ces lieux des lectures d'enfance sont présentés dans une certaine diversité, toute relative cependant. Si le narrateur déclare s'adonner au plaisir de lire dans la salle à manger qui est tranquille le matin, dans sa chambre aux heures chaudes du jour, et le soir, dans un labyrinthe constitué par une charmille après le goûter, il reste en permanence dans des lieux familiaux et pour cela familiers. Ces lieux proustiens de l'enfance possèdent, également, quelque chose d'un chronotope à la manière de Bakhtine, dans la mesure où le lieu de lecture est lié à un moment particulier :

les lectures de l'enfance dont le souvenir doit rester pour chacun de nous une bénédiction. [...] ce qu'elles laissent surtout en nous, c'est l'image des lieux et des jours où nous les avons faites. Je n’ai pas échappé à leur sortilège : voulant parler d'elles, j'ai parlé de tout autre chose que des livres parce que ce n'est pas d'eux qu'elles m'ont parlét ${ }^{\circ}$.

38. M. Petit, L'Art de lire ou comment résister à l'adversité, Paris, Belin, 2008.

39. M. Proust, "Journées de lecture», dans Contre Sainte-Beuve, ouvr. cité, p. I6I.

40. Ibid., p. I7I. 
De fait, l'importance du lieu des lectures d'enfance n'apparaît qu'avec le temps, c'est le passage du temps qui effectue un déplacement de l'ordre du paradoxe entre l'intérêt premier pour les livres mêmes et celui qui va concerner ensuite les conditions matérielles de la lecture. Alors que, dans le passé, le livre lu est la seule chose qui emporte le lecteur et lui importe, avec le temps, c'est le souvenir des circonstances mêmes de la lecture qui a pris de l'intérêt :

s'il nous arrive encore aujourd'hui de feuilleter ces livres d'autrefois, ce n'est plus que comme les seuls calendriers que nous ayons gardés des jours enfuis, et avec l'espoir de voir reflétés sur leurs pages les demeures et les étangs qui n'existent plus ${ }^{4 \mathrm{I}}$.

Le lieu de la lecture apparaît ainsi comme la concrétisation d'un temps; il définit une époque de la vie personnelle. Cette caractéristique s'explique bien entendu par une vision du monde dans laquelle lieux et temporalité sont fondamentaux. Que les moments des lectures d'enfance aient une grande importance chez un écrivain qui se présentera ensuite «à la recherche du temps perdu» n'est pas pour surprendre; que les lieux des lectures en aient également autant pour un écrivain qui organise deux parties de son roman encyclopédique autour du "pays», dont il envisage d'abord seulement le nom («Nom de pays : le nom») avant d'évoquer ensuite une réalité plus concrète ("Nom de pays : le pays") a également toute sa logique.

D'une certaine importance si l'on se réfere à la longueur du passage qui leur est dédié, les lieux de la lecture apparaissent aussi comme un espace relié à d'autres. Celui qui est pleinement évoqué est «le site merveilleux, différent du reste du monde, et au cœur duquel nous voudrions qu'ils nous fissent pénétrer ${ }^{42}$ ", à savoir un lieu du monde retenu un peu par hasard que les auteurs magnifient par leur génie. Cette dimension spatiale à l'intérieur même de la lecture ouvre sur la métaphore, banale somme toute, du parcours, de la promenade ou de la flânerie, qui va être utilisée par Proust, lequel l'adapte et la spécifie selon ses goûts :

Un peu du bonheur qu'on éprouve à se promener dans une ville comme Beaune [...] on ressent encore un peu de ce bonheur à errer au milieu d'une tragédie de Racine ou d'un volume de Saint Simon ${ }^{43}$.

4I. Ibid., p. I60.

42. Ibid., p. 177 .

43. Ibid., p. I9I. Cette thématique est reprise plus loin : «cette impression si exaltante qui fait ressembler certaines "journées de lecture” à des journées de flâneries à Venise, sur la piazetta par exemple» (p. 194). 
Enfin, les différents lieux sont en interaction. C'est ainsi que l'espace réel permet à l'énergie intérieure suscitée par la lecture de s'exprimer, une fois que le livre a été lu. On touche ici à la dimension physique, corporelle de la lecture présentée comme un moment d'échanges entre les énergies psychique et physique :

le livre était fini. Il fallait arrêter la course éperdue des yeux et de la voix qui suivait sans bruit, s'arrêtant seulement pour reprendre haleine, dans un soupir profond. Alors, afin de donner aux tumultes depuis trop longtemps déchaînés en moi pour pouvoir se calmer ainsi d'autres mouvements à diriger, je me levais, je me mettais à marcher le long de mon lit, les yeux fixés à quelque point qu’on aurait vainement cherché dans la chambre ou dehors, car il n'était situé qu'à une distance d'âme, une de ces distances qui ne se mesurent pas par mètres et par lieues, comme les autres $[\ldots]^{44}$

Trois espaces se trouvent ainsi emboîtés les uns dans les autres : l'espace réel et relativement large de la chambre, l'autre espace réel, mais plus limité, de la page, et un dernier espace, d'une tout autre configuration et d'un autre statut, l'espace intérieur que laisse apercevoir la formule «distance d'âme».

$\grave{A}$ propos de ce texte qui donne une grande importance aux lieux des lectures d'enfance et qui s'inscrit très nettement dans les conceptions de la lecture subjective, je ferai trois remarques.

\section{Des lectures dans le monde familial contre les lectures en bibliothèque?}

La première de celles-ci concerne la signification de ce texte, que j'interrogerai maintenant en situation. Je l'ai signalé, «Journées de lecture» est devenu la préface à la traduction de Sésame et les lys, un ouvrage fait de deux conférences de John Ruskin, "Directeur de conscience de son temps» et "Professeur de goût" selon les termes mêmes de Proust (dans un article paru en I9oo à la mort du penseur anglais $\left.{ }^{45}\right)$. Or, cette préface est très nettement sous le signe du paradoxe, de manière triple. En plus de celui que j’ai déjà mentionné, un deuxième apparait dès la première phrase, laquelle suggère un renversement de la croyance selon laquelle lire n'est pas vivre:

44. Ibid., p. I70.

45. "John Ruskin", article paru dans La Chronique des Arts et de la curiosité, repris dans M. Proust, Journées de lecture, ouvr. cité, p. 67-69. 
Il n'y a peut-être pas de jours de notre enfance que nous n'ayons si pleinement vécus que ceux que nous avons cru laisser sans les vivre, ceux que nous avons passés avec un livre préféré ${ }^{4}$.

Mais la modalité paradoxale s'étend à l'ensemble du propos puisque ce texte préfaciel qui devrait être de présentation est, de fait, un texte de discussion : Proust manifeste un certain nombre de désaccords avec les points de vue développés par celui qu'il introduit. Dans son livre Marcel Proust, Théories pour une esthétique ${ }^{47}$, Anne Henry souligne ces désaccords en observant que Proust utilise cette préface pour effectuer "un travail de sape», fruit d'une " animosité venimeuse qui sous-tend tant de propositions insinuant que Ruskin n'a jamais eu toute sa tête ${ }^{48}$ ». La préface serait ainsi un règlement de comptes que Proust mènerait envers Ruskin, lui permettant d'avancer vers la conquête de sa propre esthétique.

En quoi cette analyse d'un positionnement de Proust "contre Ruskin» nous intéresse-t-elle ici? Tout simplement dans la mesure où le texte de Ruskin que préface Proust, Sésame et les lys, développe des positions sur la lecture, dans une perspective qui n'est pas fondamentalement centrée sur le lecteur individuel comme celle de Proust, mais qui relève de ce qu'Anne Henry appelle le «socialisme esthétique» du penseur anglais. Les conférences de Ruskin, en effet, font partie d'un combat pour la culture, dans un monde où les intérêts et les biens matériels occupent de plus en plus de place. Critiquant l'indifférence pour les arts des élites anglaises de son temps, Ruskin en vient à prôner la fondation de bibliothèques publiques royales. Pour Anne Henry, le début de la préface, c'est-à-dire le récit des journées de lecture, est l'une des attaques stratégiques ménagées par Proust contre Ruskin : pour elle, «cette enfance de petite bourgeoisie provinciale catholique dans laquelle il jouera à se glisser (en lorgnant du côté de Barrès)» sert de "brouillage de l'esprit critique présenté comme nécessaire entrée en matière ${ }^{49}$ ".

Ainsi, les magnifiques «lectures d'enfance» insérées dans des lieux dont elles permettent de conserver des souvenirs émerveillés s'inscrivent contre des propositions "plus terre à terre», comme le dit Anne Henry, de développement de la culture par la création de bibliothèques. Si les lectures d'enfance en des lieux ainsi devenus mémorables sont écrites contre les lectures dans les bibliothèques publiques, l'interrogation de ce récit d'une «Journée de lecture» à la lumière d'autres écrits de Proust nous conduira à découvrir un autre

46. "Journées de lecture», Contre Sainte-Beuve, ouvr. cité, p. I6o.

47. A. Henry, Marcel Proust. Théories pour une esthétique, Paris, Klincsieck, I98I.

48. Ibid., p. 206.

49. Ibid., p. 207-208. 
positionnement. En effet, le développement sur les lectures d'enfance possède une forme d'avant-texte dans Jean Santeuil ${ }^{\circ}$ et il sera repris de manière particulière dans Du côté de chez Swann ${ }^{\text {厂I }}$.

Qu'en est-il des lieux de lecture dans ces textes?

\section{«Journées de lecture» entre les «Lectures ${ }^{52}$ » dans Jean Santeuil et les «Lectures au jardin" dans Du côté de chez Swann}

Dans le roman inachevé et dans le premier volume de La Recherche du temps perdu, plusieurs des éléments que l'on a vu développés dans «Journées de lecture» se retrouvent. C'est particulièrement le cas dans Jean Santeuil. Ainsi, le fragment intitulé «Lectures» et celui qui lui fait suite dans l'édition de Pierre Clarac portent tous deux sur la lecture du Capitaine Fracasse, comme dans le texte publié en 1905 : exactement comme dans celui-ci, il est d'abord question des conditions matérielles de la lecture et des lieux où elle est possible, avant le développement d'analyses sur le rapport du jeune lecteur au roman de Gautier. La thématique de la lecture comme refuge dans une quiétude heureuse se retrouve également : «lever les yeux de son livre ${ }^{53}$ » équivaut ici, à travers un identique regard sur la chambre, celle-ci étant pourtant évoquée comme un décor un peu différent, à goûter le bien-être et la tranquillité dans l'isolement. On retrouve, en outre, les dérangements causés par les membres de la famille, même s'il ne s'agit pas des mêmes personnes, des cousins bruyants dans Jean Santeuil, un oncle dans "Journées de lecture». Le fragment du roman inachevé et le récit qui sert d'entrée en matière à "Journées de lecture» notent aussi la nécessité, pour le héros et narrateur, de changer de lieu pour préserver sa lecture, même si, ici encore, ce ne sont pas les mêmes lieux dont il est question. La préface à Sésame et les lys apparaît donc, dans sa partie initiale, comme une réécriture des deux fragments de Jean Santeuil, auxquels a été ajoutée une réflexion sur la lecture comme discussion du point de vue de Ruskin.

50. M. Proust, Jean Santeuil, précédé de Les Plaisirs et les Jours, éd. P. Clarac, avec la collaboration de Y. Sandre, Paris, Gallimard, coll. «Bibliothèque de la Pléiade», 197I.

5I. À la Recherche du temps perdu, t. I, éd. sous la dir. de J.-Y. Tadié, Paris, Gallimard, I987, coll. "Bibliothèque de la Pléiade», p. 82-87 pour "Lecture au jardin, sous le marronnier", suivi de "L'état de ma conscience pendant la lecture», "Les personnages de roman », "Paysages évoqués par les livres», "Mes rêves de voyage et d'amour".

52. M. Proust, Jean Santeuil, ouvr. cité, p. 309-313, suivi de Le Capitaine Fracasse, p. 313-316.

53. Pour reprendre la formule de Y. Bonnefoy dans Revue de psychanalyse, $\mathrm{n}^{\circ} 37, \mathrm{I} 988$, consacrée à la lecture. 
Le passage de Du côté de chez Swann qui a pour intertitre "Lecture au jardin sous le marronnier» dans l'un des "résumés » de ce volume ${ }^{54}$, transforme la réflexion. S'il est également une réécriture des textes précédents dans la mesure où il en reprend explicitement certains motifs comme la lecture dans la chambre, les empêchements produits par différents bruits, ou par un parent trop bien attentionné, lequel cause un changement de lieu de lecture, ou encore les réflexions sur le rapport du jeune lecteur aux personnages, il diffère passablement des textes précédents, tout d'abord par son intégration dans un roman qui a évoqué dans son premier mouvement la chambre d'enfant, à côté des autres chambres dont se souvient le narrateur à demi éveillé, et par l'évocation d'une première lecture dans cette chambre, sur laquelle je reviendrai. Là est sans doute la raison pour laquelle le récit de la lecture dans le roman publié en I9I3 se passe essentiellement au jardin. En outre et surtout, la célébration du monde de l'enfance conservé à travers le souvenir de la lecture disparaît comme tel au profit d'une analyse de «l'espèce d'écran diapré d'états différents» que, pendant sa lecture, "déployait simultanément [sa] conscience et qui allaient des aspirations les plus profondément cachées en (lui-même) jusqu'à la vision tout extérieure qu' [il avait], au bout du jardin, sous les yeux [... $]^{55}$ ».

C'est ainsi que le récit de la maturité créatrice de Proust présente l'intérêt de mettre en évidence la juxtaposition de différents états de la conscience lectrice qui conjoint une quête de la vérité, les émotions suscitées par l'action romanesque, fait alors apparaître «le paysage où se déroulait l'action et qui exerçait sur [sa] pensée une bien plus grande influence que l'autre», ainsi que, finalement, le paysage qu'il avait «sous les yeux quand il les levait du livre ${ }^{56}$ ». Cette diversité des états de la conscience lectrice renvoie aisément à la multiplicité des "postures du lecteur» de Dominique Bucheton ${ }^{57}$ ou des «activités de fictionnalisation» de Gérard Langlade et Nathalie Lacelle ${ }^{\varsigma 8}$.

On le voit, si, de Jean Santeuilà Du côté de chez Swann, un certain nombre de motifs concernant la lecture sont repris, la représentation proustienne en

54. Intertitre donné par le résumé dans l'édition de J.-Y. Tadié, ouvr. cité p. I524. L'édition de P. Clarac et A. Ferré, a choisi l'intertitre de «Lectures au jardin» pour son résumé (p 993). 55. À la recherche du temps perdu, éd. J.-Y. Tadié, t. I, ouvr. cité, p. 83.

56. Ibid., p. 85 .

57. D. Bucheton, "Les postures de lecture des élèves au collège ", dans P. Demougin et J.-F. Massol (coord.), Lecture privée lecture scolaire : la question de la littérature à l'école, Grenoble, CRDP de l'académie de Grenoble, 1999, "Documents, Actes et Rapports", p. I37-I5O.

58. G. Langlade et N. Lacelle, "Former les lecteurs/spectateurs par la lecture subjective des œuvres", dans J.-L. Dufays, Enseigner et apprendre la littérature aujourd'hui, pour quoi faire? Sens, utilité, évaluation, Louvain-la-Neuve, Presses universitaires de Louvain, 2007, p. 55-64. 
enrichit les significations et transforme le rapport au lieu de lecture. D'autres textes de Proust, qui ne sont nullement des variations sur le même scénario, peuvent être ajoutés pour comprendre l'ampleur et la richesse de la réflexion sur la lecture et la question des lieux de celle-ci.

\section{D'autres moments de lecture un peu différents}

J'évoquerai trois autres textes qui complexifient cette représentation de la lecture.

Dans Jean Santeuil d'abord, l'évocation des lectures d'enfance est particulière car elle apparaît en partie en un lieu différent de celui où se font les lectures évoquées dans le fragment déjà mentionné. Ce lieu qui élargit la représentation des lectures personnelles, leur donne une dimension différente, et qui va quasiment disparaître ensuite de la représentation proustienne, est l'institution scolaires9, mentionnée à travers le collège puis le lycée, nommément le lycée Henry IV. Dans les fragments du roman inachevé inscrits en ces deux lieux, l'évocation de ceux-ci n'est nullement menée comme on l'a vu pour les lieux familiers de la lecture : ni le collège, ni le lycée parisien ne font l'objet d'une quelconque description. En revanche, on peut relever quatre différences majeures avec l'évocation des lectures d'enfance, qui spécifient les lectures en lien avec l'institution scolaire telle que l'envisage Proust dans son roman inachevé.

On peut d'abord noter que ces lectures en rapport avec le collège puis le lycée portent sur des genres autres que le roman : lectures de poèmes, lectures de contes, ce qui est expressément conforme aux programmes officiels d'une époque qui ne reconnaît aucune légitimité au roman. Cependant, Leconte de Lisle remplaçant le Gautier du Capitaine Fracasse, la prégnance du discours de l'art pour l'art sur le jeune héros reste importante, même si, pour la poésie, le romantisme de Hugo et de Musset est présent également. Ensuite, dans les fragments concernant le collège comme le lycée, les lectures personnelles se trouvent toujours évoquées en rapport avec les lectures scolaires, même si ce rapport est souvent d'opposition, Jean préférant, par exemple, les poèmes de Leconte de Lisle aux classiques qui lui sont donnés à lire et qu'il devrait aimer, Racine au premier chef. En outre, ces lectures sont toujours présentées

59. À propos de la disparition de l'institution scolaire dans La Recherche du temps perdu, voir "Composition française et dissertations romanesques, quand deux "grands écrivains" représentent l'institution scolaire", dans J.-F. Massol, De l'institution scolaire de la littérature française (I870-I925), Grenoble, Ellug, 2004, p. I8I-25I. 
en relation avec des personnages d'enseignants, Rustinlor, dans le fragment intitulé «Le lycée Henry IV ${ }^{60}$ » et «Éveil de Jean à la poésie», ou M. Beulier dans celui qui est intitulé "Les étrennes de M. Beulier", ce qui ouvre sur la dernière caractéristique, à savoir que la lecture personnelle est soit commentée, dans les discussions avec Rustinlor, soit faite à haute voix et écoutée, lorsque M. Beulier lit des contes à ses élèves «Une triste veille de Noël» ou "Quelques jours avant le jour de l'An ${ }^{61}{ }$ encore. Il en est ainsi lorsque, au cours d'une répétition qu'il donne, chez lui, au jeune héros, le même professeur lit à Jean "les pages admirables où Michelet célèbre le Travail en Hercule... ${ }^{62}$ » ou des pages de Xénophon ${ }^{63}$. Dans les deux derniers cas, la lecture qui permet aux goûts personnels de s'affirmer dans une confrontation à des textes prescrits ou proposés, est liée à une relation interpersonnelle avec le professeur : s'il y a bien socialisation de la lecture et réaction personnelle à des contraintes ou des propositions, celle-ci reste limitée à une relation duelle : les autres élèves sont absents (alors qu'ils peuvent être présents par ailleurs dans les fragments mentionnés).

De manière plus générale, ne peut-on dire que les lectures d'enfance célébrées dans les pages régulièrement citées, "Journées de lecture" constituant une variation sur les pages de Jean Santeuil, sont construites contre deux autres formes de lectures? La magie des lectures personnelles dans différents lieux privés et intimes s'élèverait ainsi à la fois contre les lectures dans les bibliothèques, dont Ruskin propose un développement pour défendre l'art et la culture, et contre les lectures scolaires que les enseignants proposent en raison des missions qui leur sont confiées, mais aussi avec des points de vue divers, le maître Rustinlor du jeune Jean Santeuil n'ayant pas sur la culture scolaire le même point de vue que M. Beulier?

Deux autres textes doivent encore être pris en considération.

Dans Du côté de chez Swann, la lecture à haute voix évoquée dans les fragments concernant $M$. Beulier est bien présente également, mais c'est la mère du Narrateur, cette fois, qui porte cette forme de lecture. Et cette lecture ancrée dans la voix de la mère, apparaît, en outre, à une place importante, fondatrice. Le début du récit $\grave{A}$ la recherche du temps perdu est constitué par la scène du baiser de la mère du Narrateur, demandé, refusé, attendu et finalement obtenu.

6o. «Comme son maître Rustinlor, Jean préférait à tous les poètes Verlaine, Leconte de Lisle, et comme lui éprouvait à la lecture des classiques un morne ennui " (Jean Santeuil, ouvr. cité, p. 236).

6I. Ibid., p. 265.

62. Ibid., p. 266-267.

63. Si le héros est a priori séduit par le premier texte et déçu par le second, le point de vue que lui donne le professeur va le faire changer d'avis. 
On ne saurait oublier que cette longue séquence romanesque se termine par des lectures :

$[\ldots]$ «Voyons, puisque tu n'as pas sommeil ni ta maman non plus, ne restons pas à nous énerver, faisons quelque chose, prenons un de tes livres.» Mais je n'en avais pas. «Est-ce que tu aurais moins de plaisir si je sortais déjà les livres que ta grand-mère doit te donner pour ta fête ${ }^{64}$ ?» $[\ldots]$

Donnant lieu à un long développement où il va être question des rêvasseries qui accompagnent la lecture maternelle, cette forme première de lecture à haute voix figure ainsi le terme de la scène initiale qui ouvre La Recherche. Ici encore, le lieu de la lecture qu'est la chambre n'est pas décrit au moment où la voix de la mère fait retentir le roman de Sand, mais c'est sans doute parce qu'il apparaît auparavant de manière fragmentée en plusieurs passages.

On ne peut manquer de rappeler que cette découverte de François le Champi à travers la lecture oralisée faite par la mère, revient d'une certaine manière dans Le Temps retrouvé, construisant ainsi nettement un effet de boucle au moment où le projet du Narrateur va se trouver enfin précisément défini. Cette fois, la lecture n'a plus lieu dans la chambre, mais dans une bibliothèque, celle, privée et prestigieuse, du prince de Guermantes. Quant à la lecture proprement dite, elle se réduit à celle du titre, lequel plonge le Narrateur dans ses souvenirs d'autrefois, à savoir ceux des débuts du roman, pendant que resurgit dans le Narrateur un étranger à lui-même qui n'est autre que lui pourtant, à savoir le Narrateur enfant.

\section{Une réflexion en perspective}

Dans sa complexité d'ensemble, faite de différents textes ayant des valeurs diverses les uns par rapport aux autres, à envisager dans une dynamique qui va des avant-textes variés à $L a$ Recherche $d u$ temps perdu, l'œuvre de Proust qui propose une réflexion en mouvement sur la lecture va plus loin, pour la question qui nous a intéressés ici, que le simple enregistrement ou la simple mention de divers lieux de la lecture personnelle. N'évoquant ceux-ci avec précision que s'ils concernent les lectures d'enfance, elle leur confere une réelle importance, reprenant au moins trois fois avec variations, de Jean Santeuil à Du côté de chez Swann, ce qui devient, plus qu'un motif, le topos du lieu de lecture, et produisant de celui-ci une représentation en évolution, pour cela d'autant plus intéressante.

64. Du côté de chez Swann, éd. J.-Y. Tadié, ouvr. cité, p. 39. 
Cet enregistrement et cette description des lieux participent, bien sûr, d'une représentation plus générale des conditions de la lecture, laquelle convoque tous les sens pour leur donner un rôle dans la manifestation de la place du corps, au cours d'une activité où celui-ci reste momentanément en repos avant de récupérer des énergies que la lecture met intérieurement en mouvement. Cette présence forte des lieux de lecture permet aussi une mise en scène de l'isolement volontaire du jeune lecteur et du plaisir que celui-ci prend à une activité qui le met à l'écart pour lui permettre d'entrer dans une relation avec des personnages fictifs, des lieux imaginaires, des phrases et des textes, des auteurs. De manière plus fondamentale, ce topos se trouve lié également à la place que Proust donne à la spatialité et à la temporalité dans les représentations et les réflexions qu'il poursuit à travers son œuvre.

Dans ce motif fort proche d'un chronotope bakhtinien, la dimension de la spatialité est intéressante parce qu'elle est rarement traitée dans sa complexité par ailleurs. D'abord, le lien entre spatialité et temporalité est particulier dans les conditions mêmes de la lecture puisque la dimension temporelle de celle-ci est faite, en permanence, des souvenirs de lecture qui, en partie, sont aussi des souvenirs spatiaux. Ensuite, la diversité caractérise les lieux de lecture qui peuvent être d'une grande banalité ou marquants, mémorables parce que le lecteur s'y retrouve en lui-même de manière très intime et y construit son identité, ou facilement emportés par la néantisation du monde à laquelle la lecture procède souvent et de plus en plus, sans doute, pour un adulte devenu pleinement et ordinairement lecteur. Dans le moment même de la lecture, se met en outre en place un emboîtement de lieux de divers ordres, lesquels vont du réel à l'imaginaire, sans qu'un sens unique s'impose. En permanence présent dans cet ensemble composite de lieux qu'il ne peut occuper de manière identique, mais les percevant de façon alternée, dès qu'il veut y réfléchir, le lecteur navigue physiquement et mentalement dans un espace complexe, du réel vers le fictif et réciproquement, c'est-à-dire aussi des lieux passés remémorés vers ceux du futur dont le destin est d'être mémorisés à leur tour, cela tout aussi réciproquement.

Mais les lieux de lecture familiaux et familiers privilégiés par Proust s'inscrivent aussi dans une double opposition à d'autres lieux, dans deux perspectives différentes. Le rapport à ce lieu de lecture évident qu'est la bibliothèque reçoit deux traitements. D'un côté, la description des lectures d'enfance menées dans divers lieux familiers que propose la préface à la traduction de Sésame et les lys s'élève contre le discours ruskinien pour le développement des bibliothèques publiques. D'un autre, c'est-à-dire, cette fois, à la fin de La Recherche du temps perdu, la bibliothèque du prince de Guermantes où ne se lit qu'un titre est le lieu majeur d'un mouvement de remémoration qui s'inscrit dans l'avènement 
comme écrivain à part entière d'un Narrateur qui a longtemps cherché sa voie et qui, alors, redécouvre en lui l'enfant qu'il ne cesse pas d'être. Les lectures menées dans le cadre de l'institution scolaire qu'enregistrent certains fragments de Jean Santeuil se trouvent ainsi pleinement évoquées dans l'œuvre, mais dans un roman au rôle particulier, initial et abandonné. De ce fait, la mention du collège et du lycée conduit à souligner la disparition dont ils sont l'objet ensuite. Ces lieux institutionnels, en outre, privés d'une description détaillée, se trouvent définis tout autrement, le rapport au professeur devenant leur élément premier d'organisation.

Cette lecture inscrite dans des contextes de lieu, qui sont aussi de temps, et dans le système de double opposition que l'on vient de voir, qui se trouve définie aussi dans un rapport à quelques personnes aux rôles forts (la mère, personnage qui subsiste dans le récit terminal, les professeurs, personnages très vite oubliés), est essentiellement représentée comme la lecture purement individuelle d'un jeune homme appartenant à une bonne bourgeoisie entichée d'aristocratie, qui cherche à réaliser une vocation d'écrivain. De fait, les "journées de lecture» qui discutent le discours socialisant de Ruskin sur le nécessaire développement des bibliothèques publiques et $L a$ Recherche $d u$ temps perdu qui fait quasiment disparaître l'institution scolaire présente encore dans Jean Santeuil, installent la lecture, mise en scène dans une conjoncture précise, comme oubli du collectif, pour l'assomption du parcours individuel d'un Narrateur qui s'accomplit aussi dans une écriture fondée sur la remémoration personnelle.

À ce titre, malgré sa richesse et le bonheur de ses évocations détaillées, malgré la complexité de sa description dans la série de fragments divers que l'on a interrogés, la représentation proustienne ne peut intéresser le didacticien de la lecture littéraire comme tout enseignant de français, qu'à certaines conditions. La première est le refus des oppositions que les textes de Proust mettent en place dans la dynamique de leur évolution. Dans une perspective qui vise des élèves divers, aux origines sociales variées, qui est la nôtre, il y a lieu de reconnaître des lieux institutionnellement différents, avec des fonctionnements propres, et de penser des articulations plutôt que de mettre en évidence des oppositions, entre les différents moments et les différentes formes de la lecture que ces lieux permettent.

Par ailleurs, peu ou prou, les analyses qui précèdent montrent que les lieux de lecture, et plus généralement, le contexte de la lecture, jouent un rôle non négligeable à travers les souvenirs, mais aussi dans le présent même de la lecture, et à différents niveaux. De ce fait, même si le «liseur» du modèle picardien ne permet pas d'entrer dans l'analyse des textes, c'est une instance à ne pas négliger si l'on s'intéresse à la lecture empirique, aux expériences de lecture. Car les lieux, dans leur diversité, peuvent permettre la lecture ou 
l'embarrasser, l'empêcher, lui donner forme, sens parfois, avant d'en tirer en retour, une mémorisation et la possibilité d'une remémoration.

N'est-ce pas, d'ailleurs, parce qu'il est bien arrimé dans les lieux réels où il se trouve installé confortablement le plus souvent que le liseur peut garantir au «lu» une échappée sans risque dans l'imaginaire et dans les profondeurs intimes dont il est le moins sûr? N'est-ce pas parce qu'il est assuré de pouvoir revenir dans la réalité où il est très prosaïquement de plain-pied, parce qu’il peut à certains moments se retrouver au centre de son corps que baigne la brise ou une lumière douce ou plus vive, que le lecteur peut accepter de s'embarquer pour des voyages extraordinaires mouvementés, qui sont essentiellement mentaux? N'est-ce pas aussi grâce à eux que le lecteur peut faire l'expérience jouissive d'un dédoublement?

Finalement, la représentation aussi riche des lieux de la lecture enfantine qui est celle développée par Proust peut aussi amener le didacticien à une réflexion qui n’hésite pas à envisager des oppositions et des dépassements de celle-ci. On peut, en effet, inférer des lectures évoquées ci-dessus, quelques éléments de définition de la classe comme lieu spécifique de lecture. Pour commencer par des évidences, on peut rappeler d'abord que la grande spécificité de la classe, c'est de ne pas être un lieu familier, mais, a priori, un lieu de travail et un lieu social. C'est aussi un lieu de construction de soi de plusieurs manières, dans la sociabilité et la socialisation et non dans le retrait, dans l'élaboration de compétences et l'acquisition de connaissances et non a priori dans la rêverie à propos des histoires et des livres. Cela signifie-t-il, par exemple, que toute rêverie est absente du temps et des activités scolaires? Bien sûr que non, que ce soit en bien ou en mal. Cela implique-t-il que l'on n'ait jamais à penser la classe comme un lieu permettant à la subjectivité d'advenir? Non plus également. Une ouverture dans ce sens est, de fait, bel et bien un enjeu. Et cette dimension ne sera véritablement possible que si la classe est aussi toujours conçue en relation avec d'autres lieux que le jeune lecteur est souvent plus à même d'investir en son nom propre : la chambre, ou les autres lieux familiers, ainsi que tous les lieux où peuvent se mener des lectures personnelles et ceux où doivent se faire des lectures de travail qui ont une part plus limitée (lecture analytique) ou expressément attendue (lecture cursive) de subjectivité, les bibliothèques, de classe, d'école ou d'établissement, municipales aussi, pour des lectures complémentaires qui ont toutes leur part de dimension personnelle. On pourrait sans doute ajouter pour aujourd'hui cet espace particulier qu'est «la toile» et sa lecture en des lieux tout aussi divers et peut-être plus variés encore, où peuvent se lire des textes de toutes sortes dans un cheminement virtuel qui conjugue des parcours infinis à partir d'un outil unique et stable. 
Il faut enfin et en outre considérer que l'accent mis sur les lectures d'enfance dans l'ensemble du contexte qui est le leur est le fait d'un écrivain qui a également évoqué des discussions sur les lectures, qui a publié des critiques, des pastiches d'auteurs divers, et a même mené, sur certaines démarches de la critique littéraire, les analyses marquantes du Contre Sainte-Beuve. Ce qui justifie pleinement l'intérêt de la lecture subjective mais doit aussi nous conduire à installer cette dimension des lectures dans la perspective de lectures diversifiées, plus savantes, plus élaborées, plus réfléchies. 